\title{
ON APPROXIMATION BY SOLUTIONS OF PARTIAL DIFFERENTIAL EQUATIONS
}

BY FELIX E. BROWDER ${ }^{1}$

Communicated October 10, 1961

Introduction. In a recent note in the Bulletin [1], the writer announced some results established in [2], generalizing the theorems of Walsh and Mergelyan, on uniform approximation by solutions of elliptic differential equations of arbitrary order for which the property of uniqueness in the Cauchy problem holds. The generalization of the Walsh theorem stated in [1] was restricted to elliptic operators with constant top-order coefficients. It is the object of the present note to state some sharper results whose proofs appear in [4] among which is the complete generalization of the Walsh Theorem to elliptic operators with variable top-order coefficients. The strengthening of the technical apparatus underlying these proofs permits also the generalization of our approximation theory to some classes of nonelliptic operators, and in particular to hyperbolic operators and to the class of operators of principal normal type having strongly pseudoconvex level surfaces which have been recently studied by Hörmander [5].

In order to obtain sharper approximation theorems, we must consider domains which are more smoothly bounded than the mildly regular domains of $[2]$, namely the following:

Definition. Let $G$ be a precompact domain $N$ in $E^{n}$. Then $G$ is said to be firmly regular if for each point $x_{0}$ of bdry $(G)$ there exists a neighborhood $N$, a constant $h>0$, and a unit vector $\xi_{0},\left|\xi_{0}\right|=1$, such that the cone at $x$ given by

$$
C_{x}=\left\{y: y=x+r \xi, 0<r<h,|\xi|=1,\left|\xi-\xi_{0}\right|<h\right\}
$$

is completely contained in $G$ for each $x$ in $N \cap \bar{G}$.

The basic property of firmly regular open sets which underlies our approximation theorems is the following:

Lemma 1 (Lemma 8 of [4]). Let $G$ be an open subset of $E^{n}, G_{1} a$ firmly regular open subset with compact closure in $G, A$ a linear differential operator of order $r$ with coefficients in $C^{1}(G)$.

(a) Let $u$ be an element of the Sobolev space $W^{r-1, q}(G)$ with support in $\bar{G}_{1}$ such that $A u$ is a finite Radon measure on $\bar{G}_{1}$. (We denote the space

${ }^{1}$ Sloan Fellow. The preparation of this paper was partially supported by N.S.F. Grants G-8236 and G-19751. 
of finite Radon measures on $\bar{G}_{1}$ by $M\left(\bar{G}_{1}\right)$.) Then there exists a sequence $\left\{\Psi_{k}\right\}$ with each $\Psi_{k}$ in $C_{c}^{\infty}\left(G_{1}\right)$ such that

$$
\begin{gathered}
\Psi_{k} \rightarrow u \text { in } W^{r-1, q}\left(G_{1}\right), \\
A \Psi_{k} \rightarrow A u \text { in the weak* topology on } M\left(\bar{G}_{1}\right) .
\end{gathered}
$$

(b) Let $u$ be an element of $W^{r-1, q}(G)$ with support in $\bar{G}_{1}$ such that $A u \in L^{q}(G)$. Then there exists a sequence $\left\{\Psi_{k}\right\}$ from $C_{c}^{\infty}\left(G_{1}\right)$ such that

$$
\begin{aligned}
\Psi_{k} & \rightarrow u, \text { in } W^{r-1, q}\left(G_{1}\right), \\
A \Psi_{k} & \rightarrow A u, \text { in } L^{q}\left(G_{1}\right) .
\end{aligned}
$$

Using Lemma 8 together with a general approximation argument given in [2] and [4], we establish the following results:

Theorem 1 (Theorem 2 of [4]). Let $G$ be an open subset of $E^{n}, G_{1}$ a firmly regular open subset with compact closure in $G$. Suppose that $A$ is a linear elliptic differential operator with coefficients in $C^{1}(G)$ such that $A^{\prime}$, its formal adjoint, also has its coefficients in $C^{1}(G)$. Suppose that $G-G_{1}$ has no compact components and that $A^{\prime}$ satisfies the condition $(U)_{s}$ of uniqueness in the Cauchy problem in the small on $G$.

Let $S$ be the family of solutions $u$ in $C^{r}(G)$ of the equation $A u=0$ in $G, S_{1}$ the family of solutions $u_{1}$ in $C^{0}\left(\bar{G}_{1}\right) \cap C^{r}\left(G_{1}\right)$ of the equation $A u_{1}=0$ in $G_{1}$. Then the restrictions of $S$ to $\bar{G}_{1}$ are dense in $S_{1}$ in the uniform norm on $\bar{G}_{1}$.

TheOREM 2 (TheOREM 4 of [4]). Let $G$ be a bounded open subset of $E^{n}$ contained as a proper part of another bounded open set $G^{\prime}$. Suppose that the boundary $\Gamma$ of $G$ is a manifold of class $C^{2 m}(m \geqq 1)$ in $E^{n}$ and let $G_{1}$ be a firmly regular open subset with compact closure in $G$ such that $G^{\prime}-G$, has no compact components. Suppose that $\Gamma_{0}$ is a proper open subset of $\Gamma$ bounded by a finite number of $(n-2)$-dimensional manifolds of class $C^{2 m}$ in $\Gamma$ and such that $\Gamma_{0}$ lies on the boundary of $G^{\prime}$. Let $A$ be a regularly elliptic, differential operator of order $2 m$ on $G^{\prime}$, with coefficients in $C^{1}\left(\bar{G}^{\prime}\right)$ and such that $A^{\prime}$ has coefficients in $C^{1}\left(\bar{G}^{\prime}\right)$. Suppose that $A^{\prime}$ satisfies the condition $(U)_{s}$ of uniqueness in the Cauchy problem in the small on $G^{\prime}$.

Let $S$ be the family of solutions $u$ in $C^{2 m}(G) \cap C^{m-1}\left(G \cup \Gamma_{0}\right)$ of the equation $A u=0$ in $G$ with null Dirichlet data of order $m$ on $\Gamma_{0}, S_{1}$ the family of solutions $u_{1}$ in $C^{2 m}\left(G_{1}\right) \cap C^{0}\left(\bar{G}_{1}\right)$ of $A u_{1}=0$ in $G_{1}$. Then every $u_{1}$ in $S_{1}$ can be approximated in uniform norm on $\bar{G}_{1}$ by functions $u$ in $S$.

We remark that we have extended Theorem 2 to the class of regular elliptic boundary value problems in [3].

To state our results on the nonelliptic case in a concise form, we introduce the following definition: 
Definition. Let $A$ be a partial differential operator of order $r$ with $C^{\infty}$ coefficients on an open subset $G$ of $E^{n}, G_{1}$ and $G_{2}$ firmly regular open subsets of $G$ with $\bar{G}_{1} \subset G_{2}, \bar{G}_{2}$ compact in $G$. Then the pair $\left(G_{1}, G_{2}\right)$ is said to be an approximation pair with respect to $A$ on $G$ if the following condition holds:

(1) If $u \in \mathcal{E}^{\prime}(G)$ has its support in $\bar{G}_{2}$ and $A^{\prime} u$ has its support in $\bar{G}_{1}$, then $u$ must have its support in $\bar{G}_{1}$.

In the terminology of [2], condition (1) states that the $A^{\prime}$-envelope of $\bar{G}_{1}$ in $\mathcal{E}^{\prime}\left(\bar{G}_{2}\right)$ is the set $\bar{G}_{1}$ itself.

Theorem 3 (Theorem 6 of [4]). Let $A$ be a partial differential operator of order $r$ with coefficients in $C^{\infty}(G), G_{1}$ and $G_{2}$ firmly regular open subsets of $G$ with $\bar{G}_{1} \subset G_{2}, \bar{G}_{2}$ compact in $G$. Suppose that $\left(G_{1}, G_{2}\right)$ is an approximation pair for $A$ on $G$ in the sense of the above Definition. Suppose further for a given $q, 1<q<\infty, j \geqq 0$, that if $u$ and $A^{\prime} u$ both lie in $W_{c}^{-j, q}(G)$, then $u$ must lie in $W_{c}^{r-1-1, q}(G)$.

Let $S$ be the family of solutions $u$ of the equation $A u=0$ in $G_{2}$ with $u$ lying in $W^{i, p}\left(G_{2}\right)\left(p=q(q-1)^{-1}\right), S_{1}$ the family of solutions $u_{1}$ of $A u_{1}=0$ in $G_{1}$ with $u_{1}$ lying in $W^{i, p}\left(G_{1}\right)$. Then the restrictions of the functions of $S$ to $G_{1}$ are dense in $S_{1}$ in the norm of $W^{i, p}\left(G_{1}\right)$. In particular, if $j>n / p$, the functions of $S$ are dense in $S_{1}$ in the uniform norm on $G_{1}$.

We remark that for hyperbolic operators $A$ and for $p=2$, the hypotheses of Theorem 3 follow for pairs of domains $\left(G_{1}, G_{2}\right)$ such that $G_{2}-\bar{G}_{1}$ (or more precisely, $G-\bar{G}_{1}$ ) can be swept out by continuous families of space-like surfaces, as follows from Leray's theory of hyperbolic equations [6]. Similar conclusions for operators of principal normal type with strongly pseudo-convex level surfaces may be derived from Theorem 3 by applying the recent results of Hörmander $[5]$.

\section{BIBLIOGRAPHY}

1. F. E. Browder, Approximation in uniform norm by solutions of elliptic differential equations, Bull. Amer. Math. Soc. vol. 67 (1961) pp. 400-404.

2. - Functional analysis and partial differential equations. II, Math. Ann. (1961) to appear.

3. - Functional analysis and partial differential equations. III, to appear.

4. - Approximation by solutions of partial differential equations, to appear.

5. L. Hörmander, Differential operators with non-singular characteristics, Lecture Notes, Amer. Math. Soc. Conference on Functional Analysis, Stanford, 1961.

6. J. Leray, Hyperbolic differential equations, Lecture Notes, Institute for Advanced Study, 1953.

Massachusetts Institute of Technology 